\title{
Sum rules for total hadronic widths of light mesons and rectilineal stitch of the masses on the complex plane
}

\author{
Michał Majewski
}

November 9, 2018

\begin{abstract}
Mass formulae for light meson multiplets derived by means of exotic commutator technique are written for complex masses and considered as complex mass sum rules (CMSR). The real parts of the (CMSR) give the well known mass formulae for real masses (Gell-Mann-Okubo, Schwinger and Ideal Mixing ones) and the imaginary parts of CMSR give appropriate sum rules for the total hadronic widths - width sum rules (WSR). Most of the observed meson nonets satisfy the Schwinger mass formula (S nonets). The CMSR predict for $\mathrm{S}$ nonet that the points $(m, \Gamma)$ form the rectilinear stitch (RS) on the complex mass plane. For low-mass nonets WSR are strongly violated due to "kinematical" suppression of the particle decays, but the violation decreases as the mass icreases and disappears above $\sim 1.5 \mathrm{GeV}$. The slope $k_{s}$ of the RS is not predicted, but the data show that it is negative for all $\mathrm{S}$ nonets and its numerical values are concentrated in the vicinity of the value -0.5 . If $k_{s}$ is known for a nonet, we can evaluate "kinematical" suppressions of its individual particles. The masses and the widths of the S nonet mesons submit to some rules of ordering which matter in understanding the properties of the nonet. We give the table of the $\mathrm{S}$ nonets indicating masses, widths, mass and width orderings. We show also mass-width diagrams for them. We suggest to recognize a few multiplets as degenerate octets. In Appendix we analyze the nonets of $1^{+}$ mesons.
\end{abstract}

\section{Introduction}

Total particle width is one of its main attributes just as important as mass and discrete quantum numbers. It tells us something different than the mass and sometimes it may tell more. The widths of the particles with similar masses may differ by many orders. Then the widths first inform us which interaction strong, electromagnetic or weak is responsible for their decay.

Obviously, the total hadronic widths are not so much differentiated, but still are remarkably various. Within $S U(3)_{f}$ meson multiplet the differences are often of the same magnitude as between the masses. Thus they merit attention. 
However, as the mass formulae have been derived long ago, no relation is known between the total widths. Perhaps such situation is due to conjecture that it is not worthy to pay attention to the widths pattern of the multiplet, as the widths are in a way accidental. Indeed, selection rules and phase space may suppress more or less the decay of particular particle thus destroying any given regularity. Such an effect should be especially transparent in low-mass multiplets where e.g., for some particle two-body decays are forbidden and many-body decays are suppressed (the meson $\omega$ is a striking example). For more massive multiplets, where many decay channels are opened, we may expect better agreement. However, the prediction may be interesting in any case.

\section{Exotic commutator mass sum rules (ECMSR)}

The approach is based on the exotic commutator technique [1]. We assume that the following set of exotic commutators vanishes ([2])

$$
\left[T_{\alpha}, \frac{d^{j} T_{\beta}}{d t^{j}}\right]=0, \quad(j=1,2,3, \ldots)
$$

where the $T$ are $S U(3)_{f}$ generators, $t$ is time and $(\alpha, \beta)$ is an exotic combination of indices; that means that $T_{\alpha}, T_{\beta}$ are chosen such that operator $\left[T_{\alpha}, T_{\beta}\right]$ does not belong to the octet representation (we use the combination changing strangeness by two). Putting

$$
\frac{d T}{d t}=i[H, T]
$$

and using the infinite momentum approximation for the one-particle hamiltonian $[3$

$$
H=\sqrt{m^{2}+p^{2}} \simeq p^{2}+\frac{m^{2}}{2 p}+O\left(\frac{1}{p^{2}}\right),
$$

we transform eqs. (11) into the following system (cf [2])

$$
\begin{gathered}
{\left[T_{\alpha},\left[\hat{m^{2}}, T_{\beta}\right]\right]=0,} \\
{\left[T_{\alpha},\left[\hat{m^{2}},\left[\hat{m^{2}}, T_{\beta}\right]\right]\right]=0,} \\
{\left[T_{\alpha},\left[\hat{m^{2}},\left[\hat{m^{2}},\left[\hat{m^{2}}, T_{\beta}\right]\right]\right]\right]=0,}
\end{gathered}
$$

where $\hat{m}^{2}$ is the squared-mass operator. Taking matrix elements of these operator equations between one-particle octet states and saturating the products of flavoured operators with one-particle intermediate octet states, we get the system of linear equations for the matrix elements $\left\langle x_{8}\left|\left(\hat{m}^{2}\right)^{j}\right| x_{8}\right\rangle(j=1,2,3, \ldots)$, where $\left|x_{8}\right\rangle$ is the isoscalar octet state. Solving this system, we find

$$
\left\langle x_{8}\left|\left(\hat{m}^{2}\right)^{j}\right| x_{8}\right\rangle=\frac{1}{3} a^{j}+\frac{2}{3} b^{j} \quad(j=1,2,3, \ldots) .
$$


Here $a$ is an isovector particle mass squared and

$$
b=2 K-a,
$$

with $K$ being the mass squared of the isodublet particle. Notice that

$$
\left\langle x_{8}\left|\hat{m^{2}}\right| x_{8}\right\rangle=\frac{1}{3} a+\frac{2}{3} b=x_{8}
$$

is the Gell-Mann-Okubo mass.

Let us discuss a nonet. Introduce isoscalar physical states $\left|x_{1}\right\rangle,\left|x_{2}\right\rangle$ which are linear combinations of the exact symmetry octet $\left|x_{8}\right\rangle$ and singlet $\left|x_{0}\right\rangle$ states. Consequently, we can write

$$
\left|x_{8}\right\rangle=l_{1}\left|x_{1}\right\rangle+l_{2}\left|x_{2}\right\rangle,
$$

where $l_{1}, l_{2}$ are real and

$$
l_{1}^{2}+l_{2}^{2}=1 .
$$

Using (6), we transform eqs. (3) into the system of equations

$$
l_{1}^{2} z_{1}^{j}+l_{2}^{2} z_{2}^{j}=\frac{1}{3} a^{j}+\frac{2}{3} b^{j}, \quad(j=1,2,3, \ldots)
$$

where $x_{1}, x_{2}$ are masses squared of the physical isoscalar mesons (we choose $\left.x_{1}<x_{2}\right)$.

Eqs. (7) and (8) are basic for the further investigation. They are considered as linear equations for the unknown coefficients $l_{1}^{2}, l_{2}^{2}$ and they are examined by the gradually enlarging number of eqs. (8). This enables us to obtain by a uniform procedure the known results of the broken $S U(3)_{f}$ symmetry. In the succeeding steps, beginning from the first of eq. (8), we find the known mass formulae: Gell-Mann - Okubo (GMO), Schwinger (S) and Ideal Mixing (IM). We also find the mixing angle in each of these cases. Let us discuss the procedure in more detail.

If we consider only eq. (7) and the first of eq. (8) (one exotic commutator), we find

$$
l_{1}^{2}=\frac{x_{2}-x_{8}}{x_{2}-x_{1}}, \quad l_{2}^{2}=\frac{x_{8}-x_{1}}{x_{2}-x_{1}} .
$$

Hence, for an octet $\left(l_{1}^{2}=1, l_{2}^{2}=0\right)$ we get GMO mass formula

$$
x_{1}=x_{8} .
$$

For a nonet we have $l_{1}^{2}>0, l_{2}^{2}>0$. Introducing the mixing angle $\theta^{G M O}$

$$
l_{1}^{2}=\sin ^{2} \theta^{G M O},
$$

we find

$$
z_{1} \sin ^{2} \theta^{G M O}+z_{2} \cos ^{2} \theta^{G M O}=z_{8} .
$$


This equation determines $\theta^{G M O}$ which is usually quoted as the nonet mixing angle. There is no mass formula in this case and the $\theta^{G M O}$ is determined only by the experimental masses. Such a nonet we call the GMO one.

If we consider eq. (77) and the first two of eqs. (8) (two exotic commutators), then we have three equations for two unknown quantities. Solution (9) remains true provided the masses satisfy a consistency condition. This condition is the well known $\mathrm{S}$ mass formula. In terms of the parameters $a$ and $b$ the mass formula and the coefficients $l_{1}^{2}, l_{2}^{2}$ are:

$$
\begin{gathered}
\left(a-x_{1}\right)\left(a-x_{2}\right)+2\left(b-x_{1}\right)\left(b-x_{2}\right)=0, \\
l_{1}^{2}=\frac{1}{3} \frac{\left(x_{2}-a\right)+2\left(x_{2}-b\right)}{x_{2}-x_{1}}, \\
l_{2}^{2}=\frac{1}{3} \frac{\left(a-x_{1}\right)+2\left(b-x_{1}\right)}{x_{2}-x_{1}} .
\end{gathered}
$$

These equations define another mixing angle, the $\theta^{S c h}$ one. Such a nonet we call an $\mathrm{S}$ one.

If we consider three of eqs. (8) (three exotic comutators), then we get one more consistency condition

$$
a\left(a-x_{1}\right)\left(a-x_{2}\right)+2 b\left(b-x_{1}\right)\left(b-x_{2}\right)=0,
$$

and consequently we obtain the IM nonet:

$$
z_{1}=a, \quad z_{2}=b \quad \text { and } \quad l_{1}^{2}=\frac{1}{3}, \quad l_{2}^{2}=\frac{2}{3} .
$$

The signs of $l_{1}, l_{2}$ are not determined. Ideally mixed isoscalar states arise, if $l_{1} l_{2}<0$.

It is now obvious that any additional eq. (8) (for $\mathrm{j}=4,5, \ldots$ ) is an identity.

The exotic commutator approach is the only known way to derive the ideally mixed states from something else. Alternatively they are postulated.

The current shape of the nonet mass formula

$$
\left(x_{2}-x_{8}\right)\left(x_{8}-x_{1}\right)=\gamma^{2},
$$

is obtained by diagonalizing the mass matrix

$$
\left[\begin{array}{lr}
x_{0} & \gamma \\
\gamma & x_{8}
\end{array}\right]
$$

and eliminating $x_{0}$, which is the mass squared of the $S U(3)_{f}$ singlet. The GMO mass $x_{8}$ is known for all - GMO, S and IM nonets. The mixing parameter $\gamma$ is undetermined for the GMO nonet, but it is known for the S nonet. It can be calculated from the mass formula (13) by the substitutions

$$
a=x_{8}-\frac{2}{3}(b-a),
$$




$$
b=x_{8}+\frac{1}{3}(b-a)
$$

and observation that

$$
b-a=2(K-a) .
$$

The calculation confirms "Schwinger Ansatz":

$$
\gamma^{2}=\frac{8}{9}(K-a)^{2}
$$

Data show (or at least suggest) that, with one exception of pseudoscalar mesons, all nonets comply with this expression. So they are the $S$ nonets.

The pseudoscalar mesons $\pi, K, \eta, \eta^{\prime}$ form the only recognized GMO nonet. Its experimental masses are described by eq. (18) with mixing parameter

$$
\gamma^{2}=\frac{2}{9}(K-a)^{2}
$$

The $\mathrm{S}$ nonets are not much different from IM ones, but none of them is strictly ideal (see Tab. 1 for an explicite comparision).

For the IM nonet not only $\gamma^{2}$ is determined (eq. (22) ), but also $x_{0}$ can be calculated:

$$
x_{0}=\frac{1}{3}(2 a+b) .
$$

\section{Sum rules for complex masses (CMSR)}

\subsection{Complex mass operator}

We assume that eqs. (8) may be considered for complex mass squared. We choose the complex mass operator in the form

$$
\hat{m}_{c}^{2}=\hat{m}^{2}-i \hat{m} \hat{\Gamma}
$$

where $\hat{m}$ and $\hat{\Gamma}$ are hermitean and commute. This operator can be diagonalized and has orthogonal eigenfunctions. That follows from the observation that the operators

$$
\frac{1}{2}\left(\hat{m}_{c}^{2}+\hat{m}_{c}^{2}\right)=\hat{m}^{2} \quad \text { and } \quad \frac{i}{2}\left(\hat{m}_{c}^{2}-\hat{m}_{c}^{2}\right)=\hat{m} \hat{\Gamma}
$$

are hermitean and commute.

We use the notation

$$
a_{c}=a-i \alpha, \quad K_{c}=K-i \kappa, \quad z_{1}=x_{1}-i y_{1}, \quad z_{2}=x_{2}-i y_{2}
$$

for the complex masses of the physical particles, where

$$
a=m_{a}^{2}, \quad K=m_{K}^{2}, \quad x_{1}=m_{1}^{2}, \quad x_{2}=m_{2}^{2}
$$

and

$$
\alpha=m_{a} \Gamma_{a}, \quad \kappa=m_{K} \Gamma_{K}, \quad y_{1}=m_{1} \Gamma_{1}, \quad y_{2}=m_{2} \Gamma_{2}
$$


For subsidiary states with complex masses

$$
z_{8}=\frac{1}{3} a_{c}+\frac{2}{3} b_{c}, \quad b_{c}=2 K_{c}-a_{c}
$$

we use a similar notation:

$$
z_{8}=x_{8}-i y_{8}, \quad b_{c}=b-i \beta .
$$

The parameters $\alpha, \kappa, y_{1}, y_{2}$ are positive, as they refere to real particles. Below it will be seen that $y_{8}$ is also positive. This concerns the observed values as well as the predicted ones. The question about positivity of $\beta$ is not so simple. It will be seen further that the predicted value of $\beta$ is positive for all known $\mathrm{S}$ nonets, but as

$$
\beta=2 \kappa-\alpha,
$$

it may happen that $\beta<0$ for observed values of $\alpha$ and $\kappa$. That will indicate a relative suppression of the $K$-meson decay.

It is convenient to introduce the subsidiary widths $\Gamma_{8}$ and $\Gamma_{b}$ :

$$
y_{8}=m_{8} \Gamma_{8}, \quad \beta=m_{b} \Gamma_{b} .
$$

For complex masses also the coefficients $l_{1}, l_{2}$ (6) are complex and in eqs. (77), (8) the $l^{2} \mathrm{~s}$ are replaced by the $|l|^{2} \mathrm{~s}$.

Below we show that the real parts of the masses (25) obey the usual mass formulae. For the imaginary parts there arise some new relations. We call them "width sum rules" (WSR) invoking the name "mass sum rules" (MSR) used sometimes for the mass formulae.

\subsection{Gell-Mann - Okubo nonet}

From eq. (7) and the first of eqs. (8) (for $\mathrm{j}=1$ ) we find

$$
\left|l_{1}\right|^{2}=\frac{x_{2}-x_{8}}{x_{2}-x_{1}}, \quad\left|l_{2}\right|^{2}=\frac{x_{8}-x_{1}}{x_{2}-x_{1}}
$$

and

$$
\left|l_{1}\right|^{2} y_{1}+\left|l_{2}\right|^{2} y_{2}=y_{8} \text {. }
$$

$\left|l_{i}\right|^{2}$ determines the mixing angle which depends only on the masses and is not affected by the widths. Eq. (35) shows that $y_{8}$ is positive.

It is seen from (34) that

$$
x_{1}<x_{8}<x_{2} .
$$

Therefore,

$$
\tilde{x}_{1}=x_{8}-x_{1}, \quad \tilde{x}_{2}=x_{2}-x_{8}
$$

are positive. Introducing also

$$
\tilde{y}_{1}=y_{8}-y_{1}, \quad \tilde{y}_{2}=y_{2}-y_{8},
$$

we can write (35) in the form

$$
\frac{\tilde{y}_{2}}{\tilde{x}_{2}}=\frac{\tilde{y}_{1}}{\tilde{x}_{1}} .
$$




\subsection{Schwinger nonet}

Consider eq. (7) and the first two of eqs. (87) (for $\mathrm{j}=1,2$ ). Using for complex masses eq. (18) with $\gamma^{2}$ given by (22), we find for its real part

$$
\tilde{x}_{1} \tilde{x}_{2}-\tilde{y}_{1} \tilde{y}_{2}=\frac{2}{9}\left[(b-a)^{2}-(\beta-\alpha)^{2}\right]
$$

and for the imaginary one

$$
\tilde{x}_{1} \tilde{y}_{2}+\tilde{x}_{2} \tilde{y}_{1}=\frac{4}{9}(b-a)(\beta-\alpha) .
$$

From eqs. (39) and (41) we find

$$
\begin{aligned}
& \tilde{y}_{1}=\frac{2}{9} \frac{(b-a)(\beta-\alpha)}{\tilde{x}_{2}}, \\
& \tilde{y}_{2}=\frac{2}{9} \frac{(b-a)(\beta-\alpha)}{\tilde{x}_{1}} .
\end{aligned}
$$

Substituting (42) and (43) into (40) we get a quadratic equation for the product $\tilde{x}_{1} \tilde{x}_{2}$ :

$$
\left(\tilde{x}_{1} \tilde{x}_{2}\right)^{2}-\frac{2}{9}\left[(b-a)^{2}-(\beta-\alpha)^{2}\right]\left(\tilde{x}_{1} \tilde{x}_{2}\right)-\left(\frac{2}{9}\right)^{2}(b-a)^{2}(\beta-\alpha)^{2}=0 .
$$

The solution

$$
\tilde{x}_{1} \tilde{x}_{2}=\frac{2}{9}(b-a)^{2}
$$

is just the Schwinger mass formula. It can be written in the form (13). The widths of the particles do not contribute to the mass formula. The second root $\tilde{x}_{1} \tilde{x}_{2}=-\frac{2}{9}(\beta-\alpha)^{2}$ is rejected as negative.

As $\tilde{x}_{1}, \tilde{x}_{2}$ and $(b-a)$ are positive, it follows from (42) and (43) that $\tilde{y}_{1}, \tilde{y}_{2}$ and $\beta-\alpha$ have the same sign. So, $y_{1}$ and $y_{2}$ lie on opposite sides of $y_{8}$.

Multiplying (42) by (43) and using (45), we find the formula

$$
\tilde{y}_{1} \tilde{y}_{2}=\frac{2}{9}(\beta-\alpha)^{2}
$$

which is the analogue of the Schwinger mass formula.

Finally, we notice that (cf eq. (21))

$$
\beta-\alpha=2(\kappa-\alpha)
$$

and that WSR (46) can be written in the form ( $\mathrm{cf}$ (13))

$$
\left(\alpha-y_{1}\right)\left(\alpha-y_{2}\right)+2\left(\beta-y_{1}\right)\left(\beta-y_{2}\right)=0 .
$$




\subsection{Ideal nonet}

If we use eq. (7) and the first three of eqs. (8) (for $j=1,2,3$ ), we obtain eqs. (17) for complex masses

$$
z_{1}=a_{c}, \quad z_{2}=b_{c}, \quad \text { and } \quad\left|l_{1}\right|^{2}=\frac{1}{3}, \quad\left|l_{2}\right|^{2}=\frac{2}{3} .
$$

Hence,

$$
x_{1}=a, \quad x_{2}=b, \quad \Gamma_{1}=\Gamma_{a}, \quad \Gamma_{2}=\Gamma_{b} .
$$

Note that eq. (17) and three eqs. (8) give four real conditions and three imaginary ones. The first two of the real conditions determine $\left|l_{1}\right|^{2},\left|l_{2}\right|^{2}$, the remaining two give ideal values for $x_{1}, x_{2}$. For calculating $\Gamma_{1}, \Gamma_{2}$ we have three imaginary equations. However, for ideal masses the ideal $\Gamma_{1}, \Gamma_{2}$ follow from the first two eqs. (8) and the third equation does not change the result.

\section{The rectilineal stitch of the masses on the complex plane}

The formulae (34), (45) and (49) for the real parts of the complex masses are identical with the corresponding formulae for the real ones. So, the conditions of flavour-symmetry breaking which operate in CMSR well reproduce data on the masses.

We assumed from the beginning that also in the WSR (35), (46) and (49) flavour-symmetry breaking factors are correctly taken into account. However, this does not mean that they have to describe well the real data. The particle widths depend also on non-flavour factors which violate the WSR. Let us call them for brevity "kinematical", although they may include other effects. Among "kinematical" factors the main role is played by strictly kinematical ones - the phase-space volume and conservation lows. These factors may considerable disturb the widths of the low-mass particles, but the higher is mass, the weaker is their influence due to opening of new decay-channels. Therefore, violation of the WSR would be significant in low-mass nonets and would weaken for more massive ones. The data reveal such a tendency. It is, therefore, likely that our initial assumption is true (the flavour symmetry breaking is really well described). Then violation of the WSR is a measure of the "kinematical" violation. However, it is difficult to evaluate on the basis of WSR the size of the violation and attribute it to definite particles of the nonet, because the sum rules do not include the "triangulation point". One can make the "kinematical" breaking transparent combining WSR with the mass formula.

Eqs. (42) and (43) connect the real and imaginary parts of the masses of the S nonet. By means of eq. (45), we obtain very simple result (cf (39)):

$$
\frac{\tilde{y}_{2}}{\tilde{x}_{2}}=\frac{\tilde{y}_{1}}{\tilde{x}_{1}}=\frac{\beta-\alpha}{b-a}=k_{s} .
$$


The points $(a, \alpha),(K, \kappa),\left(x_{1}, y_{1}\right),\left(x_{2}, y_{2}\right),\left(x_{8}, y_{8}\right),(b, \beta)$ lie on a straight line in the plane $\left(m^{2}, m \Gamma\right)$. Also the points $\left(m_{a}, \Gamma_{a}\right),\left(m_{K}, \Gamma_{K}\right)$ etc. lie on a straight line with the same slope in the plane $(m, \Gamma)$. The slope $k_{s}$ is indefinite.

So, the masses form a rectilineal stitch (RS) on the complex plane.

Eqs. (51) ultimately explain the nature of violation of the WSR of the S nonet. If the slope of the stitch $k_{s} \neq 0$ (true for all of $\mathrm{S}$ nonets), then the WSR and the S mass formula are equivalent and satisfy the same conditions of the broken flavour-symmetry. Therefore, flavour breaking does not operate and violation of the WSR of the S nonet is "kinematical".

Reversing the argument, one can say that rectilineality of the stitch is a result of flavour-symmetry breaking which identically influence the mass formula and the WSR.

\section{Ordering rules}

The expressions (14), (15) derived for an $\mathrm{S}$ nonet do not guarantee positivity of $\left|l_{1}\right|^{2},\left|l_{2}\right|^{2}$. This is required additionally. The requirement introduces further restrictions on the masses which take the form of an ordering rule. There are two allowed mass orderings of the S nonet (cf [6]) implying also two distinct inequalities between some masses and two different ranges of the mixing angle $\theta^{\text {Sch }}$ :

$$
\begin{array}{ccc}
a<x_{1}<b<x_{2} ; & 2 K<x_{1}+x_{2} ; & \theta^{S c h}>\theta^{i d}, \\
x_{1}<a<x_{2}<b ; & 2 K>x_{1}+x_{2} ; & \theta^{S c h}<\theta^{i d},
\end{array}
$$

where $\theta^{i d}=35.26^{\circ}$ is the ideal mixing angle. The mass ordering rules follow from eqs. (13), (14) and (15)) under conditions $\left|l_{1}\right|^{2}>0,\left|l_{2}\right|^{2}>0$. The inequalities for the masses follow from the ordering and the relation $a+b=2 K$. The inequalities for $\theta^{S c h}$ follow from the ordering and the equation

$$
\tan ^{2} \theta^{S c h}=\frac{\left|l_{1}\right|^{2}}{\left|l_{2}\right|^{2}} .
$$

To obtain the ordering rules for widths we combine

$$
\begin{aligned}
& y_{8}=\alpha+\frac{2}{3}(\beta-\alpha), \\
& y_{8}=\beta-\frac{1}{3}(\beta-\alpha)
\end{aligned}
$$

with eqs. (42) and (43) and observe that for the nonet (52)

$$
\tilde{x}_{1}<\frac{2}{3}(b-a), \quad \tilde{x}_{2}>\frac{1}{3}(b-a)
$$

and for the nonet (53)

$$
\tilde{x}_{1}>\frac{2}{3}(b-a), \quad \tilde{x}_{2}<\frac{1}{3}(b-a) .
$$


We thus find two possible width orderings for each mass ordering: for the rule (52) we find

$$
\alpha<y_{1}<\beta<y_{2} \quad \text { or } \quad \alpha>y_{1}>\beta>y_{2}
$$

and for the rule (53)

$$
y_{1}<\alpha<y_{2}<\beta \quad \text { or } \quad y_{1}>\alpha>y_{2}>\beta .
$$

For the GMO nonet the only restriction on the masses (eq. (36)) follows from requirement of positivity of $|l|^{2} \mathrm{~s}$. Therefore, besides the rules (52) and (53) there are also possible the inequalities

$$
a<x_{1}<x_{2}<b \quad \text { and } \quad x_{1}<a<b<x_{2},
$$

where the conditions (52), (53) for $K$ and $\theta^{G M O}$ do not hold. In particular, the equality $\theta^{G M O}=\theta^{\text {id }}$ is possible for a nonideal nonet: $x_{1} \neq a, x_{2} \neq b$. Therefore, for such a nonet the value of $\theta^{G M O}$ would not yield a criterion of ideality. However, we do not know such a nonet as yet.

\section{Bird's eye view on nonet data}

Tab. 1 collects data on seven $\mathrm{S}$ nonets ordered by increasing $K$. To make the data more transparent the physical masses and widths quoted from PDG 44 are supplemented with the calculated values of $m_{b}=\sqrt{b}$ and $\Gamma_{b}=\frac{\beta}{m_{b}}$ ("mass" and "width" of the ideal state $s \bar{s}$ ). We also indicate for each nonet the mixing angle $\theta$ as well as the mass and width ordering.

In the IM nonet the numbers from neighbouring columns 3 and 4 as well as 5 and 6 would be equal. As they are not, the nonets are not ideal. Instead, all of them are the $\mathrm{S}$ nonets. That can be checked by saturating the Schwinger mass sum rule with the masses lying within the bounds of experimental error. These masses define mixing angle $\theta^{\text {Sch }}$. On the other hand, we can calculate $\theta^{G M O}$ using mean experimental values of the masses. The mixing angle $\theta$ (we assume $0<\theta<\frac{\pi}{2}$ ) quoted in Tab. 1 is in most cases the $\theta^{G M O}$ one. The calculated $\theta$ often have big errors. In several cases we cut the errors using restriction (52) or (53). Observe, that for the orderings (52) or (53) which are allowed by Schwinger mass formula, the requirements formulated for $\theta^{S c h}$ are also valid for $\theta^{G M O}$ and that these angles are not far removed from each other in the vicinity of $\theta^{i d}$.

Some further remarks are in order.

Two known pseudoscalar multiplets are not included in Tab. 1. The first is the nonet $\pi, K, \eta, \eta^{\prime}$ which is not the $\mathrm{S}$ one and, besides, has no hadronic decays (except of $\eta^{\prime}$ ). The second is the multiplet (nonet?) $\pi(1300), K(1460)$, $\eta(1295), \eta(1440)$ for which we cannot establish even the mass ordering, owing to big errors of $\pi(1300)$ and $K(1460)$ masses.

The masses of the unphysical states $K_{A}\left(1^{++}\right)$and $K_{B}\left(1^{+-}\right)$are required to satisfy the $\mathrm{S}$ nonet constraints. That makes their mixing angles the $\theta^{S c h}$ ones 
by definition. The states $K_{A}$ and $K_{B}$ are superpositions of the physical states $K_{1}(1270), K_{1}(1400)$ and therefore the masses of $K_{A}$ and $K_{B}$ must satisfy an additional condition imposed by mixing. These three constraints prove to be very restrictive, and we find that the values of $K_{A}$ and $K_{B}$ obeying them are contained within narrow intervals which are comparable with error ranges of the $K_{1}(1270)$ and $K_{1}(1400)$ masses. Also we find that values of $a_{1}$-meson mass allowed by these constraints cover only part of the range of experimental data. For the details of the procedure, see the Appendix.

The values of $\beta$ (see eq. (32) ) for the nonets $1^{--}$and $1^{++}$, calculated from the data on $a$ - and $K$-meson widths, come down deeply into the region of negative values (Tab. 1 ), while the sum rules predict $\beta>y_{2}$. In both cases we accept the width of $a$-meson as a measure of "normal" (unsuppressed) width suitable for the nonet (even if the error of the $a_{1}$-meson width is so big). In the $1^{--}$ nonet, $\beta<0$ explicitly indicates a deficiency of $K^{*}$-meson width. In the nonet $1^{++}$, the scope of the calculated $\beta$ comprise negative values as well as positive ones. The negative values may be explained by the big error of the $a_{1}$ width, without invoking a deficit of the $K_{A}$ width. In that case (if the width of $K_{A}$ were really not reduced) the $a_{1}$ width would be close to the lower limit of the experimental value. Similar remarks can also be made for other multiplets. However, that and other disagreements between prediction and data are better seen from mass-width diagrams.

Fig. 1 exhibits mass-width diagrams of the same S nonets. On each diagram, besides the points $(m, \Gamma)$ representing the observed mesons, we draw the straight line crossing two or more of the points. With one exception of $K_{3}(1780)$ (the data on $\eta_{2}(1870)$ we consider uncertain), the line is drawn in such a way that there are no experimental points lying above it. That follows from the guess that deviations from the RS occur only downward. The guess itself reflects observation that suppressing-decay mechanisms are well known and frequent, while nothing certain is known about enhancing-decay mechanisms. For some of the nonets we also show dominating channels of the decay. We wonder whether the straight lines can be identified with the RS.

The most striking feature of these diagrams is the negative slope of the straight lines for all nonets (heavier particles have smaller width).

Let us discuss the diagrams of some nonets in more detail. Begin from the nonets $1^{--}, 2^{++}$where we have the most complete data. We draw the straight lines over the points $(\rho, \Phi)$ for $1^{--}$and $\left(f_{2}, f_{2}^{\prime}\right)$ for $2^{++}$. The dominating channels of the hadronic decays of these particles $\rho \rightarrow \pi \pi, \Phi \rightarrow K \bar{K}, f_{2} \rightarrow \pi \pi, f_{2}^{\prime} \rightarrow K \bar{K}$ are J,I,P,C,G,S - allowed, but in the case of $\Phi \rightarrow K \bar{K}$ the phase-space is small and the width would be relatively reduced. Therefore, if we want to identify these lines with the RS, we should remember that $1^{--}$line is steeper. From the Fig. 1 we read off $k_{s}\left(1^{--}\right)=-0.56$ and $k_{s}\left(2^{++}\right)=-0.44$.

For the nonet $3^{--}$we draw the line over the points $\omega_{3}, \rho_{3}, \Phi_{3}$. Each of the particles has a phase-space large enough for many decay channels. Apparently, at these energies the number of opened channels is sufficient for the particles to satisfy the RS equation. From the Fig. 1 we read off $k_{s}\left(3^{--}\right)=-0.43$. 
It is, therefore, likely that the slopes of RS are similar for the nonets $1^{--}$, $2^{++}, 3^{--}$and are concentrated inside the interval $k_{s}=-0.5 \div-0.4$.

The diagrams of $2^{-+}$and $0^{++}$nonets do not conflict with this observation. We do not appeal to them, as they suffer from uncertain data on the isoscalar mesons $\left(2^{-+}\right)$or from an uncertain nonet assignment $\left(0^{++}\right)$.

The situation is different with $1^{+}$nonets. For the mesons of both $1^{+-}$and $1^{++}$nonets the decays into $\pi \pi$ and $K \bar{K}$ are kinematically forbidden. Their twoparticle decays producing heavy-meson $(\rho, \omega$, etc. $)$ and many-particle decays are mostly more or less suppressed by the phase space. For the $1^{++}$nonet where we have more data, the straight line shown on Fig. 1 crosses the points $a_{1}$ and $f_{1}(1420)$. Perhaps the decays of the $a_{1}$-meson $\left(a_{1} \rightarrow(\pi \rho)_{\text {Swave }}\right.$ etc. $)$ may be considered unsuppressed, as this particle has many decay channels opened and a huge width (although the error is extremally big); but the decays $f_{1}(1420) \rightarrow$ $K \bar{K} \pi, K \bar{K}^{*}(892)+$ c.c. are clearly suppressed. So the line is based on the particle which has kinematically suppressed decays. Therefore, we cannot accept it as the RS one. A similar situation holds for the $1^{+-}$nonet.

\section{Degenerate octet}

The ninth meson does not mix with the octet, if $l_{2}^{2}=0$. Then, $x_{1}=x_{8}, l_{1}^{2}=1$ and $x_{2}$ is arbitrary. It follows also from (45) that $b=a$ and thus $K=a=x_{8}$. Then, from (42) we find $\tilde{y}_{1}=0$, from (46) $\beta=\alpha$ and thus $\beta=\kappa=y_{8}$. We see that the restrictions put by the first two exotic commutators (10) on the octet states provide their full degeneration. This result remains unchanged, if further exotic commutators are included.

Tab. 2 shows two $1^{--}$multiplets and the $4^{++}$multiplet which can be understood as degenerate octets. The degeneracy of these octets is clearly seen from the data on the masses. It is confirmed by data on the widths.

On the other hand the data on the widths confirm the suggestion that at these energies the WSR are well satisfied.

\section{Summary and discussion}

The descripton of unstable particle quantum states via attributing to them a complex mass is under study from various points of view for a long time (see e.g., [5] and references therein). We make an attempt, for the first time, to apply the idea of a complex mass for the derivation of extended mass formulae within the early proposed algebraic approach 2 2 based on exotic commutators of the $S U(3)_{f}$ charge operators and their time derivatives in the infinite momentum frame system. The new sum rules including hadronic widths of the resonances are compared with the data for ten meson multiplets and the gains and problems encountered are of a certain interest and may also serve as a starting point for a further investigation.

The real parts of the complex mass sum rules (CMSR) give relations between 
Table 1: Schwinger nonets of mesons ( $m$ and $\Gamma$ in $\mathrm{MeV}$ ). Nonets, ordered by increasing $m_{K}$, are described in three rows containing: masses; widths; mixing angle, mass and width ordering. Subscripts $a, K, 1,2$ denote isotriplet, isodublet and isoscalar states. $m_{b}=\sqrt{b}$ and $\Gamma_{b}=\frac{\beta}{m_{b}}$ are calculated. In ordering rules $a$, $b, x_{1}, x_{2}$ are masses squared and $\alpha, \beta, y_{1}, y_{2}$ are products of mass and width. Mixing angle $\theta$ is the $\theta^{G M O}$, except of $1^{++}, 1^{+-}$where it is $\theta^{S c h} . K_{A}, K_{B}$ are unphysical nonet states (Appendix). Notations and data quoted from RPP [4].

\begin{tabular}{|c|c|c|c|c|c|}
\hline$J^{P C}$ & $\begin{array}{l}m_{K} \\
\Gamma_{K}\end{array}$ & $\begin{array}{c}m_{a} \\
\Gamma_{a} \\
\end{array}$ & $\begin{array}{l}m_{1} \\
\Gamma_{1}\end{array}$ & $\begin{array}{c}m_{b} \\
\Gamma_{b}\end{array}$ & $\begin{array}{l}m_{2} \\
\Gamma_{2}\end{array}$ \\
\hline particles & $\theta^{G M O}$ & \multicolumn{2}{|c|}{ mass ordering } & \multicolumn{2}{|c|}{ width ordering } \\
\hline $1^{--}$ & \multirow[t]{2}{*}{$893.88 \pm 0.26$} & \multirow{2}{*}{$769.3 \pm 0.8$} & \multirow[t]{2}{*}{$782.57 \pm 0.12$} & \multirow[t]{2}{*}{$\overline{1001.7 \pm 1.1}$} & \multirow[t]{2}{*}{$1019.456 \pm 0.020$} \\
\hline$\bullet \rho(770)$ & & & & & \\
\hline $\begin{array}{l}\bullet K^{*}(892) \\
\bullet \omega(782)\end{array}$ & $50.7 \pm 0.8$ & $149.2 \pm 0.7$ & $8.44 \pm 0.09$ & $-24.8 \pm 2.1$ & $4.26 \pm 0.05$ \\
\hline • $\Phi(1020)$ & $(39.28 \pm 0.16)^{\circ}$ & \multicolumn{2}{|c|}{$a<x_{1}<b<x_{2}$} & \multicolumn{2}{|c|}{$\alpha>y_{1}>\beta>y_{2}$} \\
\hline $1^{+-}$ & \multirow{2}{*}{$1324 \pm 8$} & \multirow{2}{*}{$\overline{1229.5 \pm 3.2}$} & \multirow[t]{2}{*}{$1170 \pm 20$} & \multirow{2}{*}{$1414 \pm 9$} & \multirow[t]{2}{*}{$1386 \pm 19$} \\
\hline$\bullet b_{1}(1235)$ & & & & & \\
\hline $\begin{array}{l}\bullet K_{B} \\
\bullet h_{1}(1170)\end{array}$ & $135 \pm 17$ & $142 \pm 9$ & \multirow[t]{2}{*}{$360 \pm 40$} & $130 \pm 40$ & $91 \pm 30$ \\
\hline • $h_{1}(1380)$ & $0 \div 35.26^{\circ}$ & $x_{1}<a<x_{2}<b$ & & \multicolumn{2}{|c|}{$y_{1}>\alpha>y_{2}>\beta$} \\
\hline $1^{++}$ & $1340 \pm 8$ & $1230 \pm 40$ & $1281.9 \pm 0.6$ & $1420 \pm 12$ & $1426.3 \pm 1.1$ \\
\hline $\begin{array}{l}\text { - } a_{1}(1260) \\
\text { - } K_{A} \\
\text { - } f_{1}(1285)\end{array}$ & $134 \pm 16$ & $250 \div 600$ & $24.0 \pm 1.2$ & $-447 \div 89$ & $55.5 \pm 2.9$ \\
\hline - $f_{1}(1420)$ & $35.26^{\circ} \div 41.00^{\circ}$ & $a<x_{1}$ & $<b<x_{2}$ & $\alpha>$ ? & $>\beta>y_{2}$ \\
\hline 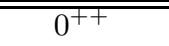 & $\overline{1412 \pm 6}$ & $984.7 \pm 1.2$ & $980 \pm 10$ & $\overline{1737 \pm 11}$ & $\overline{1713 \pm 6}$ \\
\hline $\begin{array}{l}\bullet a_{0}(980) \\
\bullet K_{0}(1430) \\
\bullet f_{0}(980)\end{array}$ & $294 \pm 23$ & $50 \div 100$ & $40 \div 100$ & $380 \div 490$ & $125 \pm 10$ \\
\hline - $f_{0}(1710)$ & $(33.5 \pm 2.0)^{\circ}$ & $x_{1}<c$ & $<x_{2}<b$ & $y_{1}>$ & $>y_{2}>\beta$ \\
\hline $2^{++}$ & $1429.0 \pm 1.4$ & $1318.0 \pm 0.6$ & $1275.4 \pm 1.2$ & $1532.0 \pm 3.1$ & $1525 \pm 5$ \\
\hline $\begin{array}{l}\bullet a_{2}(1320) \\
\bullet K_{2}^{*}(1430) \\
\bullet f_{2}(1270)\end{array}$ & $103.8 \pm 4.0$ & $107 \pm 5$ & $185.1_{-2.6}^{+3.4}$ & $101.5 \pm 11.9$ & $76 \pm 10$ \\
\hline$\bullet f_{2}^{\prime}(1525)$ & $\left(30.67_{-1.72}^{+1.56}\right)^{\circ}$ & $x_{1}<0$ & $<x_{2}<b$ & $y_{1}>$ & $>y_{2}>\beta$ \\
\hline $2^{-+}$ & $1773 \pm 8$ & $1670 \pm 20$ & $1617 \pm 5$ & $1870 \pm 33$ & $1842 \pm 8$ \\
\hline $\begin{array}{c}\bullet \pi_{2}(1670) \\
\bullet K_{2}(1770) \\
\eta_{2}(1645)\end{array}$ & $186 \pm 14$ & $259 \pm 10$ & $181 \pm 11$ & $122 \pm 49$ & $225 \pm 14$ \\
\hline$\eta_{2}(1870)$ & $0 \div 35.26^{\circ}$ & $x_{1}<0$ & $<x_{2}<b$ & $y_{1}>$ & $>y_{2}>\beta$ \\
\hline $3^{--}$ & $1776 \pm 7$ & $1691 \pm 5$ & $1667 \pm 4$ & $1857 \pm 11$ & $1854 \pm 7$ \\
\hline $\begin{array}{l}\bullet \rho_{3}(1690) \\
\bullet K_{3}^{*}(1780) \\
\bullet \omega_{3}(1670)\end{array}$ & $159 \pm 21$ & $161 \pm 10$ & $168 \pm 10$ & $158 \pm 53$ & $87_{-23}^{+28}$ \\
\hline$\bullet \Phi_{3}(1850)$ & $\left(32.0_{-7.5}^{+3,3}\right)^{\circ}$ & $x_{1}<c$ & $x_{2}<b$ & $y_{1}>$ & $>y_{2}>\beta$ \\
\hline
\end{tabular}



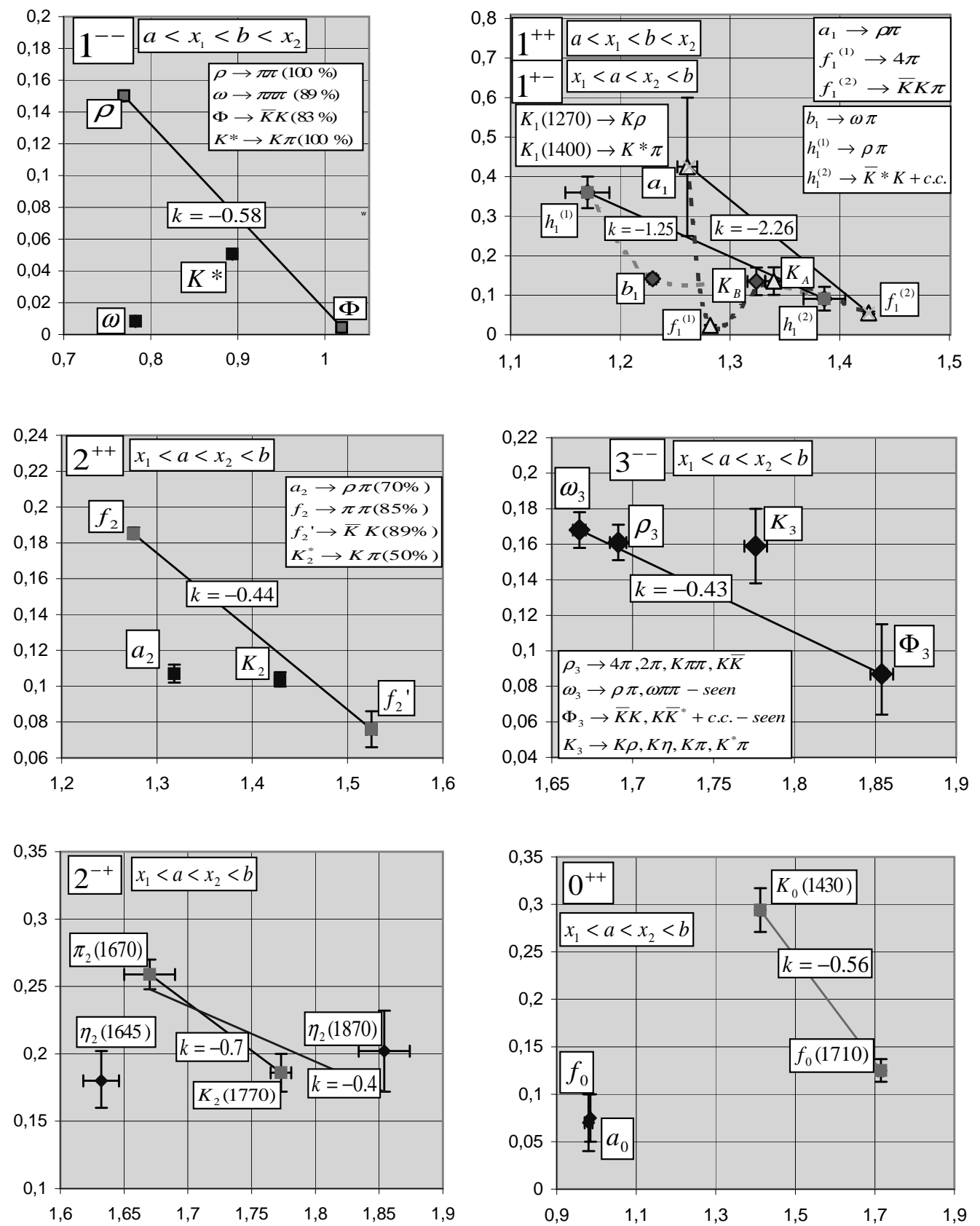

Figure 1: Mass-width diagrams of the $\mathrm{S}$ nonets. On the axes $\mathrm{m}$ and $\Gamma$ in $\mathrm{GeV}$. Shown dominating decays of some particles. Full straight lines crossing two or more points are supposed to be nearest by RS of the nonet. For $3^{--}$and $2^{++}$ they are expected to lie along the RS. For $1^{--}$small phase-space pushes down the observed point $\Phi$. For $1^{+}$nonets the straight lines cannot be identified with RS. 
Table 2: Degenerate octets ( $m$ and $\Gamma$ in $\mathrm{MeV}$ [4])

\begin{tabular}{|c|c|c|c|}
\hline$J^{P C}$ & $\begin{array}{c}m_{a} \\
\Gamma_{a} \\
\end{array}$ & $\begin{array}{l}m_{K} \\
\Gamma_{K}\end{array}$ & $\begin{array}{l}m_{1} \\
\Gamma_{1} \\
\end{array}$ \\
\hline $\begin{array}{l}1^{--} \\
\bullet \rho(1450) \\
\bullet K^{*}(1410) \\
\bullet \omega(1420) \\
\end{array}$ & $\begin{array}{l}1465 \pm 25 \\
310 \pm 60 \\
\end{array}$ & $\begin{array}{l}1414 \pm 15 \\
232 \pm 21 \\
\end{array}$ & $\begin{array}{l}1419 \pm 31 \\
174 \pm 60 \\
\end{array}$ \\
\hline $\begin{array}{l}1^{--} \\
\bullet \rho(1700) \\
\bullet K^{*}(1680) \\
\bullet \omega(1650) \\
\bullet \Phi(1680)\end{array}$ & $\begin{array}{l}1700 \pm 20 \\
240 \pm 60\end{array}$ & $\begin{array}{l}1717 \pm 27 \\
322 \pm 110\end{array}$ & $\begin{array}{rr}1649 \pm 24, & 1680 \pm 20 \\
220 \pm 35, & 150 \pm 50\end{array}$ \\
\hline $\begin{aligned} & 4^{++} \\
\bullet & a_{4}(2040) \\
\bullet & K_{4}(2045) \\
\bullet & f_{4}(2050)\end{aligned}$ & $\begin{array}{l}2011 \pm 13 \\
360 \pm 40\end{array}$ & $\begin{array}{l}2045 \pm 9 \\
198 \pm 30\end{array}$ & $\begin{array}{l}2025 \pm 8 \\
194 \pm 13\end{array}$ \\
\hline
\end{tabular}

the real parts of the complex mass squared which are identical with the well known mass formulae of the broken flavour symmetry for real masses.

The CMSR predict two possible kinds of octet: Gell-Mann - Okubo (with masses satisfying the GMO mass formula) and degenerate. So, the latter is obtained not only for exact symmetry but also under the CMSR constraints. The CMSR predict width degeneration of the mass degenerate octet particles. The data shown in Tab. 2 demonstrate that two $1^{--}$multiplets and the $4^{++}$ one are degenerate octets. The degeneration is seen not only from the masses, but also from the widths.

The CMSR predict three kinds of nonets: Gell-Mann - Okubo, Schwinger (S) and ideally mixed (IM). All the observed nonets having hadronic widths are $\mathrm{S}$ ones. As the $\mathrm{S}$ nonet well describes the masses, we may think that it correctly takes into account the flavour breaking factors.

The $\mathrm{S}$ nonet mesons are submitted to definite mass ordering. There are two allowed orderings: $x_{1}<a<x_{2}<b$ and $a<x_{1}<b<x_{2}$ (for the notations, see Sect. 2). The mass ordering decides whether the mixing angle of the nonet is smaller or bigger than $\theta^{i d}$. It also decides whether $2 K$ is bigger or smaller than $x_{1}+x_{2}$. Thus the mass ordering is a relation characterizing nonet; likewise the mixing angle characterize it.

The imaginary parts of the CMSR give relations between the imaginary parts of the complex mass squared. These relations - the width sum rules (WSR) of GMO, S and IM types - connect the total widths of the nonet mesons. WSR have shapes identical with the mass formulae, but, in contrast, they are not satisfied by data in general. The reason is an extra reduction of the widths of the individual particles by "kinematical" factors (we mean not only strictly kinematical factors, like phase-space and E,J,I,S,P,C,G - conservation, but also 
all other unflavour ones). This violation diminishes along with growth of the mean nonet mass and disappears at about $1.5 \mathrm{GeV}$.

In the $\mathrm{S}$ nonets the ordering rules are in force also for the imaginary parts of the masses. They are correlated with the mass ordering rules, but also are not satisfied by the observed widths of the low-mass nonets.

Tab. 1 collects seven $\mathrm{S}$ nonets. For each of them are shown masses, widths, mass and width ordering, and mixing angle. Deviations of the isoscalar mesons from ideal values are apparently seen.

For the $\mathrm{S}$ nonet CMSR predict a linear relation between the masses and the widths of all particles. Hence, the complex masses form a rectilineal stitch (RS) on the complex plane with a slope $k_{s}$ depending of the nonet. As $k_{s} \neq 0$, the Schwinger WSR is equivalent to the $\mathrm{S}$ mass formula and complies with the conditions breaking flavour symmetry. Therefore, violation of the WSR cannot be a result of the flavour symmetry breaking and consequently violation of the WSR for the $S$ nonet is "kinematical".

To construct the RS of a nonet we need the particles decaying in the "kinematically" unsuppressed processes. We can do that for a nonet having masses above $1.5 \mathrm{GeV}$ where many channels of the decay are oppened and WSR are satisfied. $3^{--}$is such a nonet. Also for a low mass nonet the RS can be determined approximately, if there exist two particles with "kinematically" unsuppressed dominating decays. The nonets $1^{--}$and $2^{++}$are examples. Fig. 1 demonstrates mass-width diagrams of seven nonets. For the nonets $1^{--}$and $2^{++}$the RS is constructed as the highest lying straight line crossing two experimental points $(m, \Gamma)$ of the diagram. For the $1^{+}$nonets such a line cannot be identified with the RS, because of lack of two particles decaying in "kinematically" unsuppressed reaction.

In the cases where $k_{s}$ is known we can evaluate the "kinematical" suppression of a particle as the difference between predicted (lying on RS) and observed value of the width.

The rectilineality of the stitch and the stitch itself result from flavoursymmetry breaking (for an exactly symmetric multiplet there would be only one point), but the slope of the stitch $k_{s}$ is undetermined. Data show that $k_{s}$ is negative for all nonets. They also suggest that the slopes are not much different from each other and are concentrated somewhere in the region $k_{s} \approx-0.5 \div-0.4$. The origin of such behaviour of $\Gamma(m)$ is unknown. We can only remark that it resembles the behaviour of the strong coupling constant $\alpha_{s}$, also decreasing with icreasing mass and being flavour independent.

Let us go back to the idea with which we began this paper. Hadron widths do not influence the mass formula of broken flavour symmetry and the mixing angle of the nonet. Therefore, one could once discover them and describe the nonet. That is the reason why hadron spectroscopy could completely ignore the data on the total widths of the particles. However, hadrons do have finite widths and hadron spectroscopy should obligatorily describe them. Moreover, the widths are considerable and may be important for description of the multiplet as a whole, not only as attributes of individual particles. But where is a trace of that? CMSR introduce the widths into our scope. They predict not only the WSR 
connecting the total widths of the nonet mesons, but also the RS being the result of the interplay between real and imaginary parts of the CMSR. That is the place where we can expect something new. The RS is such a relation characterizing all $\mathrm{S}$ nonets; perhaps the slope $k_{s}$ characterizes the nonet individually.

Much attention is devoted nowadays to the existence of glueballs. Search for these states requires detailed information on the multiplets. Glueball cannot be discovered by analysing the properties of a single particle. Even more, such a discovery would not be convincing. The glueball state with nonexotic quantum numbers $J^{P C}$ should mix with isoscalar $q \bar{q}$ states. Therefore, the way to identify the glueball is to look for meson decouplets including three isoscalar physical mesons and investigate them 6. Sum rules for complex masses may be useful in such analysis. Investigating of the sum rules for decouplets is in progress.

\section{Acknowledgments}

The author thanks Profs. S.B.Gerasimov, P.Kosiński, V.A.Meshcheryakov for valuable discussions and Prof. W.Tkaczyk, Dr K.Smoliński, Dr J.Olejniczak for help in computer operations.

\section{Appendix: $1^{+}$multiplets as $\mathrm{S}$ nonets}

Below, $1^{++}$mesons are called $a_{A}, K_{A}, x_{A 1}, x_{A 2}$ (nonet $\mathrm{A}$ ) and $1^{+-}$mesons are called $a_{B}, K_{B}, x_{B 1}, x_{B 2}$ (nonet B) (cf Tab. 1). We assume that each of the nonets satisfies the Schwinger mass formula. As a function of $a$ and $K$ this formula is the equation of ellipse:

$$
3 a^{2}+8 K^{2}-8 a K+a\left(x_{1}+x_{2}\right)-4 K\left(x_{1}+x_{2}\right)+3 x_{1} x_{2}=0 .
$$

The parameters of the ellipse are determined by the masses of the isoscalar mesons $x_{1}, x_{2}$ which fix the position of the ellipse centre:

$$
a^{C r}=K^{C r}=\frac{1}{2}\left(x_{1}+x_{2}\right),
$$

and the magnitude of its axes $\mathbf{a}, \mathbf{b}$ being proportional to $\left(x_{2}-x_{1}\right)$. However, they do not influence the axes' ratio $(\mathbf{a} / \mathbf{b}=3.6)$ and the orientation of the ellipse in the plane (the angle beween big axis and obscissa is $29^{\circ}$ ). So these quantities are the same for all $\mathrm{S}$ nonets.

As the straight line $2 K=x_{1}+x_{2}$ crosses the centre of the ellipse, the mass ordering (52) or (53) decides whether $K$ lies below or above its diameter.

The physical states of the $1^{+}$mesons $K_{1}(1270)$ and $K_{1}(1400)$ are mixed states of the $K_{A}$ and $K_{B}$ :

$$
\begin{aligned}
\left|K_{1}(1270)\right\rangle & =\left|K_{A}\right\rangle \cos \Phi+\left|K_{B}\right\rangle \sin \Phi \\
\left|K_{1}(1400)\right\rangle & =-\left|K_{A}\right\rangle \sin \Phi+\left|K_{B}\right\rangle \cos \Phi
\end{aligned}
$$




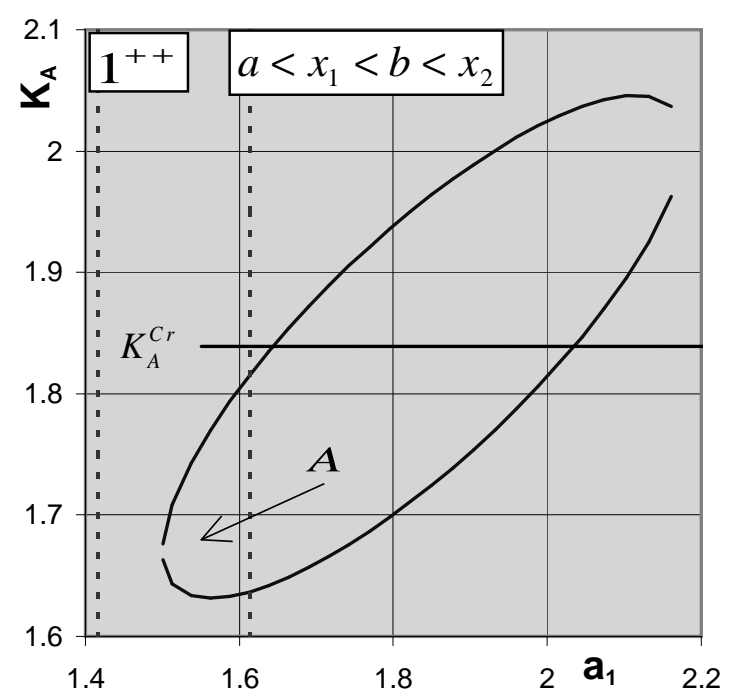

Figure 2: Ellipse A. Schwinger mass formula as function of $a_{1}$ and $K_{A}$. Dotted lines bound error corridor of $a_{1}$. Allowed $K_{A}$ belong to segment $A$ of the ellipse. They lie below diameter $K_{A}^{C r}$ as required by mass ordering. On the axes $a_{1}$ and $K_{A}$ in $\mathrm{GeV}^{2}$.

where $\Phi$ is the mixing angle 4]. For the masses squared of these mixed states we have

$$
K_{A}+K_{B}=K_{1}(1270)+K_{1}(1400) .
$$

We are looking for such values of $K_{A}$ and $K_{B}$ which satisfy this equation and eq. 62) for each nonet.

Ellipse $A$

The masses of the $1^{++}$isoscalar mesons $f_{1}(1285)$ and $f_{1}(1420)$ have negligible errors, so the ellipse $\mathrm{A}$ is precisely determined. It is shown on Fig. 2. We can see that $a_{A}$ may be assigned to the $\mathrm{S}$ nonet, only if

$$
a_{A}>(1225 M e V)^{2} .
$$

This, together with the experimental limit 4, gives

$$
(1225 \mathrm{MeV})^{2}<a_{A}<(1270 \mathrm{MeV})^{2} .
$$

From Fig. 2 we find

$$
(1277 M e V)^{2}<K_{A}<(1347 M e V)^{2} .
$$

Ellipse $B$

The mases of the $1^{+-}$isoscalar mesons $h_{1}(1190), h_{1}(1380)$ have considerable errors which influence the parameters of the ellipse (62). The Fig. 3 presents three ellipses corresponding to the mean experimental values of $x_{B 1}, x_{B 2}$ and to 


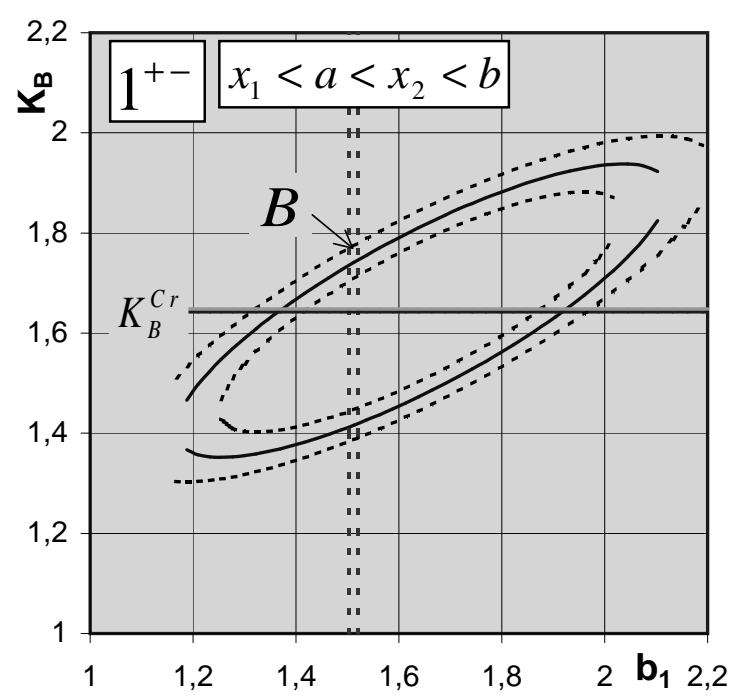

Figure 3: Ellipse B. Mass errors of $h_{1}^{(1)}, h_{1}^{(2)}$ create corridor shown between dotted ellipses. Its crossing with $b_{1}$ corridor produces solutions for $K_{B}$ above and below ellipse diameter $K_{B}^{C r}$. Choice $K_{B}>K_{B}^{C r}$ follows from mass ordering. On the axes $b_{1}$ and $K_{B}$ in $\mathrm{GeV}^{2}$.

these two combinations of their lower and uper experimental limits which give the ellipses with minimal and maximal axes. Putting for $a_{B}$ its experimental value, we find two regions of solutions for $K_{B}$. The lower solutions are rejected by the mass ordering (53) and we obtain

$$
(1307 \mathrm{MeV})^{2}<K_{B}<(1332 \mathrm{MeV})^{2} .
$$

Observe that in spite of the less accurate data on the isoscalar meson masses, the evaluation of the $K_{B}$ is more accurate than of the $K_{A}$ one.

Summing (68) and (69) we find

$$
(1828 \mathrm{MeV})^{2}<K_{A}+K_{B}<(1895 \mathrm{MeV})^{2} .
$$

On the other hand, from the data on the masses of the $K_{1}$ mesons we have

$$
(1884 M e V)^{2}<K_{1}(1270)+K_{1}(1400)<(1904 M e V)^{2} .
$$

Comparing (71) with (69), we conclude that

$$
K_{B}<\frac{1}{2}\left(K_{1}(1270)+K_{1}(1400)\right)
$$

and therefore,

$$
K_{B}<K_{A}
$$


Comparing (70) with (71), we find that the restrictions on the $K_{A^{-}}$and $K_{B^{-}}$ meson masses following from the nonet assignment are compatible with the observed mass values of $K_{1}$-mesons within the narrow interval of the sum $K_{A}+K_{B}$ :

$$
(1884 \mathrm{MeV})^{2}<K_{A}+K_{B}<(1895 \mathrm{MeV})^{2},
$$

where the upper limit of the sum is the sum of the individual upper limits of $K_{A}$ (68) and $K_{B}$ (69). Therefore, it immediately folows that adjustable values of $K_{A}$ and $K_{B}$ are:

$$
\begin{aligned}
& K_{A}=(1340 \pm 8)^{2} \mathrm{MeV}^{2} \\
& K_{B}=(1324 \pm 8)^{2} \mathrm{MeV}^{2}
\end{aligned}
$$

Returning to the ellipse $\mathrm{A}$, we find the adjustable value of $a_{A}$ :

$$
a_{A}=(1261 \pm 9)^{2} M e V^{2} .
$$

The mixing angle $\Phi$, which can be calculated from (75), (76) and the physical masses of $K_{1}$ mesons, is charged with a big error exceeding the difference $\left(\Phi^{\text {mean }}-45^{\circ}\right)$. Therefore, for evaluating $\Gamma_{A}$ and $\Gamma_{B}$ (using the physical $\Gamma_{K} \mathrm{~s}$ ) we put $\Phi=45^{\circ}$. The calculated widths are indicated in Fig. 1.

\section{References}

[1] S.Oneda, K.Terasaki Progr. Theor. Phys. Suppl. 82 (1985)

[2] M.Majewski and W.Tybor Acta Physica Polonica B15(1984) 267

[3] W.Tybor Annalen der Physik 31 (1974) 137

[4] Particle data Group Phys. Rev. D 66 (2002) 1

[5] R. de la Madrid, M.Gadella Am. J. Phys. 70 (2002) 626

[6] M.Majewski Z. Phys. C - Particles and Fields 39 (1988) 121 